\title{
Atención temprana en niños con trastornos del neurodesarrollo
}

Early care in children with neurodevelopmental disorders

\author{
Jacqueline Ponce-Meza \\ Universidad San Ignacio de Loyola, Lima, Perú. \\ Universidad Nacional Mayor de San Marcos, Lima, Perú. \\ Fundación Felipe Antonio Custer, Lima, Perú.
}

Recibido 12-01-17
Aprobado 25-03-17

Correspondencia

Email: jaynita_22@hotmail.com

\section{Citar como:}

Ponce-Meza, J. (2017). Atención temprana en niños con trastornos del neurodesarrollo. Propósitos y Representaciones, 5(1), 403 422 doi: http://dx.doi.org/10.20511/pyr2017. v5n1.154

(c) Universidad San Ignacio de Loyola, Vicerrectorado de Investigación y Desarrollo, 2017. (cc)BY-NC-ND Este artículo se distribuye bajo licencia CC BY-NC-ND 4.0 Internacional (http://creativecommons.org/licenses/by-nc-nd/4.0/). 


\section{Resumen}

El artículo analiza la importancia de la atención temprana en el desarrollo infantil, orientando una perspectiva neuropsicológica del desarrollo. El modelo de atención temprana busca hacer referencia al conjunto de intervenciones, dirigidas a la población infantil y su trabajo en conjunto con un equipo multidisciplinar. Se presenta recomendaciones para la implementación de programas que permitan la intervención temprana en los primeros años, de tal forma que se pueda optimizar el proceso de atención a los niños afectados, por las distintas alteraciones tempranas en el desarrollo y aprendizaje.

Palabras clave: Atención temprana, trastornos del neurodesarrollo, niños.

\section{Summary}

The article analyzes the importance of early care in child development, guiding a neuropsychological perspective of development. The early care model seeks to refer to the set of interventions aimed at children and their work in conjunction with a multidisciplinary team. It presents recommendations for the implementation of programs that allow early intervention in the early years, so that the process of care for the affected children can be optimized by the different early developmental and learning alterations.

Keywords: Early care, neurodevelopmental disorders, children. 


\section{Introducción}

El desarrollo integral en la infancia es fundamental para el desarrollo humano y la construcción del capital humano y social. Marcondes, Machado, Setian y Carranza (1991) indican que el concepto de desarrollo infantil no es una tarea sencilla, y varía según las referencias teóricas que se quiera adoptar y los aspectos que se quiera abordar, mencionando así que el desarrollo es el aumento de la capacidad del individuo para la ejecución de funciones cada vez más complejas.

El desarrollo infantil es un proceso dinámico, el cual se sustenta en su base biológica, psicológica y social. Es de saber que los primeros años de vida constituyen una etapa importante en el desarrollo del niño, pues se van configurando las habilidades perceptivas, motrices, cognitivas, lingüísticas y sociales que posibilitarán una equilibrada interacción con su entorno social. Este proceso es considerado como es una parte fundamental del desarrollo humano, durante los primeros años se forma la arquitectura del cerebro, a partir de la interacción entre la herencia genética y las influencias del entorno en el que vive el niño. (Shonkoff, et al., 2012), siendo el desarrollo infantil un proceso amplio y complejo, el cual incluyendo su relación con el cuidado diario y la influencia del medio ambiente sobre el mismo.

Souza (2014) el desarrollo Infantil es un proceso activo y único para cada niño, expresado por la continuidad y los cambios en las habilidades motoras, cognitivas, psicosociales y del lenguaje, con adquisiciones cada vez más complejas en las funciones de la vida diaria y en el ejercicio de su rol social. El periodo prenatal y los primeros años iniciales de la infancia son cruciales en el proceso de desarrollo, lo cual constituye la interacción de las características biopsicológicas, heredadas genéticamente, con las experiencias que ofrece el entorno. El alcance del potencial de cada niño depende del cuidado que responde a sus necesidades de desarrollo.

Entendemos, así, al desarrollo del niño como producto continuo de los procesos biológicos, psicológicos y sociales de cambio en los que éste 
resuelve situaciones cada vez más complejas, en los cuales las estructuras logradas son la base necesaria de las subsiguientes.

Esta perspectiva del desarrollo asume su multidimensionalidad, así como la indivisibilidad de los procesos biológicos, psíquicos y sociales, los que se resumen en un todo en el cual se constituye en el niño. Estos procesos están determinados por la información genética, así como por la acción del ambiente y la interacción entre ambos. La particularidad del desarrollo es lo que permite que el niño pueda adquirir en el proceso de humanización habilidades culturalmente aceptadas para desempeñarse en forma adecuada en su contexto y adaptarse cuando éste cambia.

\section{Atención temprana.}

El desarrollo de los niños durante los dos primeros años de vida es fundamental, ya que es en esta etapa que el tejido nervioso crece y madura y está, por lo tanto, más expuesto a sufrir daño. Debido a su gran plasticidad, es también en esta época cuando el niño responde más a las terapias y a los estímulos que recibe del medio ambiente. El rápido desarrollo del niño se ve influenciado por el ambiente en el cual se desarrolle. La nutrición, el cuidado y la lactancia materna afectan directamente las conexiones que se originan en el cerebro durante los primeros años (Eming \& FujimotoGomez, 2003).

Para Huthsson y Nicholl (1988) la atención temprana busca, a través de la vigilancia del desarrollo comprender todas las actividades relacionadas a la promoción del desarrollo normal y a la detección de problemas en el desarrollo, durante la atención primaria de la salud del niño. Es un proceso continuo y flexible, que incluye información de los profesionales de salud, padres, profesores y otros.

Se puede entender como atención temprana al conjunto de intervenciones infantil, entre los 0-6 años, a la familia y al entorno, que tienen por finalidad dar respuesta, lo antes posible a las necesidades transitorias o permanentes que presentan los infantes con trastornos en su desarrollo o que tienen el riesgo 
de padecerlos. Este tipo de intervención multidisciplinar es planificada y acompañada, en la intervención, por un conjunto de especialistas, que buscan resolver las necesidades del niño. (Libro Blanco de Atención Temprana, 2000)

El proceso de atención temprana debe comenzar lo antes posible, los primeros meses de vida, y ha de estar basada en una cuidadosa evaluación individual de las capacidades y de las dificultades del niño. Implantar un programa de intervención lo más tempranamente posible es muy aconsejable, incluso antes de esclarecer de manera definitiva un diagnóstico con garantías, tratando inicialmente los síntomas autísticos

La atención temprana, busca favorecer la adaptación el menor, en su entorno inmediato; en la familia, se procura brindar una atención que mejore los conocimientos y estrategias de ayuda al niño. A ello se suma un conjunto de acciones y propuestas que deben realizarse en contextos de salud, educativos o comunitarios. Este tipo de intervención reviste gran importancia por su relación con aspectos del neurodesarrollo en las primeras oportunidades de aprendizaje, y por sus aportes a la detección e intervención temprana de niños y niñas que presentan riesgos o alteraciones del desarrollo o discapacidades, entre otras. (Martínez \& Matamoros, 2010).

Los problemas en el desarrollo del niño pueden presentarse de diversas maneras: como alteraciones en el desarrollo motor, en el lenguaje, en la interacción personal-social, en lo cognitivo, etc. La mayoría de las veces existen compromisos que afectan más de una función. En este caso, el niño tiene alteraciones funcionales mixtas en su desarrollo, la presentación clínica de los niños con problemas de desarrollo varía mucho, no solo en relación al tipo de retraso, sino también en relación a la intensidad del mismo.

El cerebro del niño se encuentra en continuo desarrollo, con un crecimiento a veces vertiginoso, y sujeto a modificaciones y conexiones propias de la continua estimulación que el entorno le provee. El conocimiento del sistema nervioso nos ayudará a poder comprender los déficits que pueden aparecer 
por un desarrollo anormal del cerebro o a causa de daños en el mismo a edades tempranas. Dependiendo del momento en el que se produzcan estas anormalidades o daños (durante el embarazo, en el periodo perinatal o en el transcurso de la infancia), sus repercusiones variarán.

El neurodesarrollo puede verse como un proceso en el cual el niño participa junto a su medio ambiente, influyéndose mutuamente y evolucionando en una dirección particular, en este contexto la evaluación no solo enfoca al niño, sino también a las interacciones entre el niño, sus padres cuidadores, familia y entorno inmediato.

Para Artigas-Pallarés, Guitart, Gabau-Vila, (2013) el neurodesarrollo es un proceso evolutivo producto de la adaptación al medio, mediante pautas de comportamiento, en busca del mantenimiento de una tasa reproductiva capaz de sostener la supervivencia de la especie.

En este contexto la neuropsicología del desarrollo infantil aborda la relación entre el proceso madurativo del sistema nervioso central y la conducta durante la infancia; considera las variables de maduración, plasticidad cerebral y desarrollo durante las primeras etapas del ciclo vital, así como los trastornos que en ellos se presenta, para diseñar o adaptar modelos y estrategias de evaluación e intervención, adecuados a la población infantil. (Martínez \&Matamoros, 2010).

Los Trastornos del Neurodesarrollo son alteraciones o retrasos del desarrollo de funciones que se asocian a la maduración del Sistema Nervioso Central y que resultan en la afectación de la habilidad que tiene el SNC para recibir, procesar, almacenar y responder a la información que llega tanto del exterior como del propio organismo.

La relación existente entre la atención temprana, en los trastornos del neurodesarrollo responde a una demanda básica derivada de la identificación de un trastorno. Ante cualquier alteración del estado de salud, se genera una fuerte y comprensible presión para actuar lo más pronto posible. Ello 
contribuye a disminuir la fuerte ansiedad derivada del problema y permite abrir esperanzadoras expectativas de mejoría.

En el caso de un trastorno del neurodesarrollo, la atención temprana tiene como primer paso reducir el estrés familiar ante la incertidumbre sobre el futuro de un hijo. Posiblemente dicha repercusión justificaría por sí misma la intervención, siempre y cuando ésta no generara otros problemas en el entorno familiar. Pero al margen de estos efectos inespecíficos, existen fuertes argumentos a favor de la eficacia terapéutica de la AT. (ArtigaPallares, 2007, p.31).

La teoría de esta atención temprana se sustenta en la existencia de periodos de aprendizaje continuos, por ello las técnicas de intervención de estimulación precoz son las que facilitan el camino a desarrollar una función correcta desde el inicio, y tratan, si hay una lesión, de mitigar sus efectos. En este contexto se busca que la atención temprana deba llegar a todos los niños que presentan cualquier tipo de trastorno o alteración en su desarrollo, sea éste de tipo físico, psíquico o sensorial, o se consideren en situación de riesgo biológico o social.

Se debe tener en cuenta la importancia de una adecuada intervención temprana para amortiguar el desarrollo negativo de la función alterada. Por ejemplo, en un niño autista posiblemente tenga más sentido valorar sus relaciones sociales, en un niño con trastorno motor será mejor valorar su capacidad adaptativa en su entorno inmediato.

\section{Conclusiones}

Los diferentes programas de desarrollo infantil, siguen siendo un gran desafío para todos los involucrados, se ha visto necesario que el apoyo no solo involucra un área específica sino la de un equipo multidisciplinar. Las acciones terapeúticas ante estos tipos de trastornos requieren una alta cualificación técnica de los profesionales de atención temprana, a la que hay que añadir competencias profesionales específicas y la empatía necesaria para sintonizar con el niño y con la familia (Milla \& Mulas, 2009). En definitiva 
el apoyo que debe recibir la atención temprana forma parte de un conjunto de redes integrales necesarias para la sostenibilidad del menor, favoreciendo y haciendo la diferencia en la producción de mejoras significativas y duraderas. (McEachin, Smith \& Lovaas, 1993).

El desarrollo de una atención temprana, para la promoción de la salud comprende diferentes peculiaridades, una de ellas corresponde a las condiciones ambientales (Mello, et al. 2014).

Los diferentes estudios nos muestran evidencia significativa que define cómo intervenciones en edades tempranas tienen un efecto positivo en logros en el plano cognitivo, no cognitivo (emocional) y en los logros educativos. En este sentido a pesar de la prevalencia de esta problemática, es difícil la identificación temprana de este trastorno, ante ello sigue siendo una desafío para los profesionales y la familia. Los retrasos y desviaciones leves son difíciles de detectar.

La intervención temprana en la infancia, según los aportes de Cuervo (2009) nos definen una serie de características a seguir:

- Implementar estrategias para la detección y atención temprana de la infancia con riesgos biológicos y con riesgos sociales y retrasos en su desarrollo o alteraciones del desarrollo, esto nos permitirá disminuir las secuelas neuropsicológicas y comportamentales.

- Diseñar instrumentos y procesos para la detección y atención de los infantes con riesgos en el desarrollo, ello nos permitirá responder a las características y diferencias intra e interindividuales del desarrollo a lo largo de la infancia.

- La detección temprana de estos trastornos en la primera infancia involucra, también, una responsabilidad de la familia, los cuidadores y de los profesionales de todos los sectores educativos, de salud, comunitarios, de tal forma que se realice una intervención temprana de manera integral y en diferentes contextos. 
En definitiva es importante mencionar que la investigación en los trastornos del desarrollo es un campo con muchas áreas de aplicación y de importancia para la detección e intervención de trastornos neuropsicológicos y del desarrollo a lo largo del ciclo vital (Mustard, 2003).

En nuestro contexto Peruano, es importante acercar la evaluación e intervención a la realidad socioeconómica de las personas, el desarrollo del infante es resultado de la combinación de factores hereditarios, biológicos y físicos, pero también de factores ambientales de tipo social y cultural, esta relación en base a los principios dan por resultado una ganancia en la validez de pruebas y procedimientos de evaluación, diagnóstico y posible intervención. En todas las situaciones patológicas se tiende a resolver el problema de manera rápida, entendiéndose que de este modo los resultados van a ser mejores, sino que se aliviará la ansiedad derivada de la incertidumbre generada ante cualquier enfermedad. En el caso de los trastornos del neurodesarrollo, esta idea se acentúa todavía más.

\section{Referencias}

Artigas-Pallarés, J. (2007). Atención precoz de los trastornos del neurodesarrollo. A favor de la intervención precoz de los trastornos del neurodesarrollo. Revista de Neurología, 44(3), 31-34.

Artigas-Pallarés, J., Guitart, M., \& Gabau-Vila, E. (2013). Bases genéticas de los trastornos del neurodesarrollo. Revista de Neurología, 56(1), 2334.

Cuervo, A (2009). Importancia de la intervención temprana en la primera infancia con riesgos en el desarrollo. Perfiles Libertadores, 5, 69-75.

Eming Young, M., \& Fujimoto-Gómez. G. (2003). Desarrollo Infantil Temprano: lecciones de los programas no formales. Revista Latinoamericana de Ciencias Sociales, Niñez y Juventud, 1(1),85-123.

Grupo de Atención Temprana. (2000). Libro Blanco de Atención Temprana. Madrid: Real Patronato de Prevención y de Atención a Personas con Minusvalía. 
Marcondes, E., Machado, D.V.M., Setian, N., Carrazza, F.R. (1991). Crescimento e desenvolvimento. En: Marcondes E. (Coord.). Pediatria básica. ( $8^{\mathrm{a}}$ ed., pp. 35-62). São Paulo: Sarvier.

Martínez, Á. C., \& Matamoros, A. M. Á. (2010). Neuropsicología infantil del desarrollo: Detección e intervención de trastornos en la infancia. Revista Iberoamericana de Psicología: Ciencia y Tecnología, 3(2), 59-68.

McEachin J.J., Smith T., Lovaas O.I. (2003). Long-term outcome for children with autism who received early intensive behavioral treatment. Am J Ment Retard, 97, 359-391.

Mello, D. F. D., Henrique, N. C. P., Pancieri, L., Veríssimo, M. D. L. Ó. R., Tonete, V. L. P., \& Malone, M. (2014). Child safety from the perspective of essential needs. Revista latinoamericana de enfermagem, 22(4), 604610. doi: 10.1590/0104-1169.3651.2458

Millá, M. G., \& Mulas, F. (2009). Atención temprana y programas de intervención específica en el trastorno del espectro autista. Revista de Neurología, 48(2), 47-52.

Mustard, F. (2003). El desarrollo infantil Inicial: salud, aprendizaje y comportamiento a lo largo de la vida. Memorias. Foro Primera Infancia y Desarrollo, el De safío de la Década. Recuperado de www. redprimerainfancia.org

Shonkoff, J. P., Garner, A. S., Siegel, B. S., Dobbins, M. I., Earls, M. F., McGuinn, L., \& Committee on Early Childhood, Adoption, and Dependent Care. (2012). The lifelong effects of early childhood adversity and toxic stress. Pediatrics, 129(1), 232-246. doi: 10.1542/peds.20112663

Souza, J. M. D. (2014). Desenvolvimento infantil: análise de conceito e revisão dos diagnósticos da NANDA-I. (Tesis Doctoral). Universidade de São Paulo, São Paulo. 